\title{
Bemerkungen zu vorstehender Abhandlung von F. Bottazzi.
}

\author{
Von Wolfgang Ostwald.
}

Vorstehende Ausfübrungen sind die Antwort auf eine mehrmalige Anfrage der Redaktion der "Kolloid-Zeitschrift" an Prof. F. Bottazzi, seine von den Anschauungen der Mehrzahl der neueren Kolloidforscher vollständig abweichenden Auffassungen über die allgemeinen Eigenschaften kolloider Systeme auch den Lesern dieser Zeitschrift vorzutragen. Der Umstand, daB diese auch a. a. O. von P. Bottazzi erhobene Kritik insbesondere die Anschauungen des Verfassers dieser Zeilen betrifft, erschien vielleicht von wenige Interesse, als der Wunsch der Redaktion, ihren Lesern in möglichster Vollständigkeit die heutigen Strömungen in der Entwicklung der Kolloidchemie vorzuführen.

Angesichts der Tatsache, daB die Ausführungen von $F$. B ot tazzi Fragen der Begriffsbildung und Systematik betreffen, kann sich der Verfasser in seiner Erwiderung kurz fassen. Denn Begriffsbildung und Systematik eines Erscheinungsgebietes sind unter allen Umständen ZweckmäBigkeitsfragen, und die ZweckmäBigkeit derselben kann nur mit einem minimalen Nutzungskoeffizienten von vornherein diskutiert, wohl aber in mannigfacher Weise erwi e s e n werden. Z. B. kann sie erwiesen -werden durch die Klärung und Vereinheitlichung der Beziehungen von Erscheinungsgruppen, die vorher ohne erkennbare Verwandtschaft nebeneinander standen, ferner und insbesondere durch Anregung zu neuen Untersuchungen und damit zur Auffindung neuer bemerkenswerter Erscheinungen, durch das Beleuchten oder ins Gesichtsfeldbringen von bisher nur nebensächlich betrachteten Figenschaften und Umständen usw. Der Verfasser würde daher vielleicht am ökonomischsten verfahren, wenn er die Ausführungen resp. Begriffsbildungen und systematischen Vorschläge F. Bottazzi's sich selbst überlassen und selbst ibre nög. licherweise klärenden und befruchtenden, neue Gesichtspunkte zur Erkenntnis der Koiloide schaffenden Wirkungen ausüben ließe, -- wenn er also auf eine Diskussion überhaupt verzichtete. Zunăchst aus Be- 
dürfnis, ein Schweigen nicht als Nichtachtung der Ueberlegungen des italienischen Forschers aufgefaBt zu sehen, dann aber in der Vermutung, möglicherweise zur (aufnehmenden oder abstoBenden) Assimilation auch dieser Resultate kolloidchemischer Porschung helfen zu können, seien die folgenden Bemerkungen gemacht. SchlieBlich handelt es sich doch hier auch um Fragen von solcher Wichtigkeit und Allgemeinheit, dab jeder Kolloidinteressent an ihnen notwendigerweise beteiligt ist.

Die Ausführungen von F. Bottazzi bedeuten nichts weniger als den Vorschlagzur Rückkehrzu einer Auffassung der Kolloidchemie, wie sie etwa Th. Graham besaB, und wie sie insbesonderevordem Jahre 1895, vorderbekannten Arbeit von C. Barus und E. A. Schneider von der Mehrzahl der Forscher geteilt wurde. Im speziellen hat nach seiner. Ansicht die ganze Entwicklung der Kolloidchemie etwa innerhalb der letzten zehn Jahre, also z. B. die Ultramikroskopie, die Gallertfiltration, die Lehre von den dispersen Systemen mit ihren Folgerungen usw., gewiß für die spezielle Erkenntnis bestimmter kolloider Systeme, nichts jedoch für die Erkenntnis der allg e m e in e n Eigenschaften kolloider Systeme oder für ihre offenkundigen Beziehungen sowohl zu "echten " Lösungen als auch zu groben Dispersionen usw. beigetragen. Kolloide Lösungen "sind homogene Systeme" "nicht mehr und nicht minder als die kristalloiden Lösungen " ${ }^{1)}$ und infolgedessen „müssen wir auch auf die Annahme verzichten, daß sich zwischen Protein- oder anderen Mizellen und Lobsungen von Kristalloiden oder auch Lösungen anderer Kolloide Vorgänge abspielen können, die von der Oberflächenenergie abhängen, z. B. Erscheinungen rein mechanischer Adsorption (F. Bottazzi)". Solche "wahre ${ }^{*}$ also homogene Kolloide sind $z$. B. EiweiBlösungen und Seifen in bestimmten Zuständen usw.

Die Konsequenzen dieser Auffassung F. Bottazzi's für die heutige Kolloidchemie sind enorm. Es gibt z. B. keine Goldhydrosole resp. Goldkolloide. „Denn es gibt aber auch organische und anorganische Stoffe, die man bisher nur als mikrogranulare Suspensionen kennen gelernt hat und die man rischt in kolloide Lösungen überführen kann. Zu dieser Gruppe gehören die sogenannten Lösungen reinen Glykogens, die Suspensionen von Metallsulfiden, die sogenannten Lösungen kolloider Metalle." "Sie sind überhaupt keine kolloiden Systeme." --

1) Sperrducke von mir. Wo. O. 
"Der Dispersitătsgrad ist ke in Hauptcharakteristikum." - „DaB die Teilchen mehr oder weniger voluminös, fest oder flüssig sind, ist ein Nebenumstand und irrelevant." - "Die GröBe und die mehr oder weniger gute Sichtbarkeit unter dem Ultramikroskop ist für die Charakterisierung dieser Teilchen nur nebensächlich." Nicht der Dispersitätsgrad, sondern die "physikalisch-chemischen Eigenschaften " unterscheiden Kolloide und Suspensionen usw.

Vielleicht zeigt nichts deutlicher als diese konzentrierte Zusammenfassung einiger Hauptresultate der Ueberlegungen von $F$. B ottazzi, moglichst in den eigenen Worten des Autors, den wirklich schreienden Widerspruch derselben mit den Anschauungen, welche nach der Meinung des Schreibers die Mehrzahl der heutigen Kolloidforscher vertritt. Der große Schritt, weichen die Kolloidchemie durch die Erfindung z. B. der Ultramikroskopie getan zu haben meint, ist nach F. Bottazzi eine Täuschung, da die optische Heterogenităt völlig irrelevant ist. Der Einfluß des Dispersitätsgrades und die systematische Untersuchung des Einflusses seiner Variationen bei allen möglichen Eigenschaften ist für die Charakteristik kolloider Systeme völlig nebensächlich; der Hinweis des Schreibers auf die Wichtigkeit dieses Faktors Anfang 1907, auf den er sich beinahe etwas einbildete, alle die schönen Untersuchungen $z$. B. von The $S v e d b e r g$ und anderer über den Einflub dieser Größe bei der Brownschen Bewegung, bei der Diffusionsgeschwindigkeit, bei der Optik kolloider Metalle, bei der Adsorption und Koagulation usw. haben nach F. Bottazzi nichts für die allgemeine Charakteristik der Kolloide geliefert! Man muB es wirklich als erstaunlich bezeichnen, daB heutzutage ein Forscher die überaus große und mannigfaltige Abhängigkeit anscheinend aber auch aller "physikalisch-chemischer Eigenschaften" vom Dispersitätsgrad nicht einmal zu ken nen scheint, jedenfalls aber mit keinem Worte auf ihre Bedeutung hinweist. GewiB bestimmen die physikalisch - chemischen Eigenschaften den Charakter auch eines dispersen Systems; aber, ist es wirklich möglich heute in Abrede zu stellen, dab diese Eigenschaften weitgehend qualitativ und quantitativ variieren mit dem Dispersitätsgrad? Einschließlich selbst derartig elementarer Eigenschaften wie Dichte und Löslichkeit, Abhängigkeiten usw., die längst bekannt sind? Die Bezeichnung des fein zerteilten, makroskopisch klaren suspendierten Goldes als Kolloid ist nach F. Bottazzi falsch oder doch wenigstens irreführend. Es wăre wirklich schlimm für die heutige Kolloidchemie, wenn nicht nur ein großer Teil ihrer neueren Untersuchungen, die wir als die schönsten und interessantesten anzusehen gewohnt waren, nur "nebensāchliche " Eigenschaften der Kolloide betreffen 
würde, sondern wenn man sich etwa auch abgewöhnen müBte, rotes wässeriges Gold als Kolloid zu bezeichnen.

Im einzelnen möchte der Verfasser noch folgendes bemerken. F-B o $t$ t a $z$ z i scheint anzunehmen, daB die Charaktersierung der Kolloide als heterogene Systeme ausschließlich auf der Ultramikroskopie derselben beruht. Da manch e Eiweißlösungen usw. (keineswegs alle) in manchen Zuständen (keineswegs in allen), z. B. im Spaltultramikroskop oder in dem Ultramikroskop von A. Cotton und H. Mouton, keine optische Heteroginität erkennen lassen, "finden sie keinen Platz in Wo. Ostwald's Klassifaktion *. Dann ist also ein Gemisch von beliebig grobem Giaspulver in Kanadabalsam oder einem anderen Dispersionsmittel mit gleichem Brechungskoeffizienten kein zweiphasiges System? Und ist F. Bottazzi etwa das Verhalten von derartigen Eiweißlősungen unter einem lichtstärkeren Ultramikroskop, z. B. dem Kardioidkondensor, bereits bekannt? An welcher Stelle hat der Schreiber dieses je behauptet, dab die optische Heterogenität "das" Kriterium der Mehrphasigkeit sei? Legen nicht schon die alten Graham'schen Versuche über den Mangel an Diffundierbarkeit, über das Verhalten bei der Dialyse, über die Filtration usw. mindestens ebenso gewichtige Beweise für die „körperliche “ Heterogenität kolloider Systeme ab? Und nehmen nicht sowohl E. Raeblmann als auch H. Bechhold, jeder für sich, in Anspruch, der eine auf ultramikroskopiscbem Wege, der andere mittelst der Gallertfiltration, zum erstenmale die physische Heterogenität von EiweiBlösungen $\mathbf{n}$ a chgewiesen zu haben.

Es ist dem Verfasser auch nie eingefallen, „die kolloiden Lösungen als disperse Systeme von den ionmolekularen Lösungen zu unterscheiden ", wie F. Bottazzi sagt. Ganz im Gegenteil hat er die ionmolekularen Lösungen mit e inbezogen unter den Begriff der dispersen Systeme, ebenso wie die optisch heterogenen oder sonst nachweisbar grobdispersen Suspensionen und Emulsionen. Und zwar geschah dies, um den sich immer mehr häufenden Ueb e rgang s e r s ch e in ung e n zwischen diesen Systemklassengerecht zu werden. Auch die W'ichtigkeit dieser Gebiete und die Notwendigkeit, sie bei einer Begriffsbildung der Kolloide und veiwandter Systeme zu berücksichtigen scheint $F$. Bottazzi durchaus nicht anzuerkennen. Und wenn F. Bottazzi anführt, dab man doch nicht grundlos“ sogenannte kristalloide Lösungen als heterogen bezeichnen könne, so sei der Hinweis erlaubt, das tatsächlich genügend Gründe für diese Matbnahmen vorliegen, die er selbst kennt. So hat die Masse eines gelösten Moleküls unzweifelhaft einen anderen Wert als das Lősungs- 
mittel, wie die Zentrifugierversuche lehren. Es ist selbstverständlich auch die elektrische Beschaffenheit eine lons anders als die seines Lossungsmittels, desgleichen seine chemische Natur sprungweise verschieden von der des Losungsmittels usw. GewiB kann man in Einzelheiten differieren, was die Bewertung z. B. der neueren Versuche von The Svedberg und J.Perrin über die "körperliche Existenz der Moleküle" anbetrifft. Aber ihnen rundweg jede Beweiskraft abzusprechen, wie dies F. Bottazzi tut, wenn-er die Annahme der Heterogenităt molekulardisperser Systeme in Abrede stellt, erscheint allerdings etwas weitgehend. Hat sich doch z. B. H. Freundlich, der gewiB ein strenger Vertreter der klassischen physikalischen Chemie ist, der Anschauung des Verfassers angeschlossen, gemäB welcher auch molekulare Losungen zweckmå日ig als disperse heterogene Systeme aufzufassen sind, gieicherweise neuerdings auch $E$. Baur, und ist doch A.Smits, ein strenger Vertreter der Phasentheorie im Sinne Ro o z bo m's zu der Auffassung gelangt, daB auch allotrope Molekülgemische ein und desselben Stoffes, wie sie z. B. bei schneller Abküblung vơn Phosphor, Schwefel usw nebeneinander bestehen, auch im Sinne der Phasentheorie als mehrphasig anzusehen sind, ganz entsprechend den sog. "Isodispersoiden" des Verfassers,

Der Verfasser glaubt, daB die Hauptschwierigkeiten in der Würdigung der neueren Entwicklung der Kolloidchemie durch $F$. B ottaz $z$ i darauf beruhen, daB letzterer Forscher in hohem MaBe noch der Ansicht ist, daB der kolloide Zustand eine Eigenschaft ist, die relativ $s$ p e $z$ if i $\mathrm{s} h$ für Stoffe von bestimmter chemischer Zusammensetzung ist. Auch für ihn ist die Kolloidchemie anscheinend noch die Lehre von den kolloiden Stoffen statt die Wissenschaft von dem kolloiden Zustand in erster Annăherung beliebiger Stoffe, analog etwa der Kristallographie usw. Auf diese irrtümliche Auffassung hat der Verfasser schon so oft hingewiesen (siehe z. B. Grundriß, 2. Auflage, Seite 123), daß er sich hier nicht wiederholen mőchte. Nur das sei hier hervorgehoben, daß sich auch die Klassifikation der Kolloide selbstverständlich nicht auf besondere chemische Spezies von häufig anzutreffenden Kolloiden bezieht, sondern ebenfalls auf die verschiedenen kolloiden $Z$ u sta ande, die unter Umständen alle von ein und demselben chemischen Individuum angenommen werden können (siehe z. B. $\mathrm{H}_{2} \mathrm{O}$ an der angeführten Stelle).

Besonders großen Wert legt F. Bottazzi auf den Solvatationszustand der dispersen Phase. Der Verfasser sieht hierin keinerlei Widerspruch zu seinen eigenen Anschauungen. Ganz im Gegenteil ist er wie $F$. Bottazzi überzeugt von der großen Rolle a $u c h$ dieses 
Faktors für die allgemeine Charakteristik der dispersen Systeme. Die Annahme eines variierenden Hydratations- oder Imbibitionszustandes involviert nämlich nichts anderes als eine der vielen Möglichkeiten einer Variation der Formart der dispersen Phase im Sinne des Verfassers. Je stärker imbibiert z. B. ein Partikelchen ist, um so mehr werden sich seine physikalisch-chemischen Eigenschaften denen einer gewöhnlichen Flüssigkeit năhern und umgekehrt. Auf die große Rolle solcher Aenderungen z. B. nur infolge von Temperatur und Konzentrationsvariation hat der Verfasser selbst aber mehrfach und mit Nachdruck hingewiesen (siehe insbesondere "GrundriB", 2. Auflage); in diesem Punkte kann der Verfasser den Ausführungen von F. Bottazzi also durchaus zustimmen.

Schlieblich sei noch bemerkt, daB die Feststellung F. Bottazzi's, gemäB welcher lebendes Plasma gew $\delta$ hnlich ultramikroskopisch leer sei, keineswegs zutrifft. Die ausführlichsten und technisch vollkommensten ultramikroskopischen Untersuchungen über lebende Zellen sind wohl bisher von dem Mitarbeiter der Ze is s-Werke N. Ga id u kov angestellt worden. Dieser Forscher ist aber zu ganz anderen Resultaten gelangt (siehe seine Monographie: Ultramikroskopie usw. in der Biologie [Jena 1910], sowie Kolloid-Zeitschrift 6, 260 [1910]) und mehrere seiner lebenden Präparate hat der Verfasser selbst zu sehen Gelegenheit gehabt.

Wenn irgendwo, dann gilt für begriffliche und systematische Bemühungen der Ausspruch, daß man den Wert derselben an ihren Früchten" erkennen soll. Der Verfasser kann wirklich nicht recht glauben, daB die zu früheren Entwicklungsstadien der Kolloidchiemie zurückkehrende Auffassung $F$. Bottazzi's sich fruchtbarer erweisen wird, als die zusammenfassende Lehre von den dispersen Systemen. Er wüBte kein einziges Kolloidproblem, das durch die Auffassung $F$. Bottazzi's geklärt würde, während er im Gegensatz hierzu vom Standpunkt der Lehre 'on den dispersen Systemen unangreifbar erscheint. Wennschon also der Verfasser den Vorschlägen F. Bottazzi's keine gute Zukunft zu wünschen vermag, so bittet er wenigstens seinen Gegner ihm die freie AeuBerung dieser Bedenken nicht verübeln zu wollen. 